\title{
Temporal response profiles of serum ubiquitin C-terminal hydrolase-L1 and the 145-kDa alpha II-spectrin breakdown product after severe traumatic brain injury in children
}

\author{
Ryan R. Metzger, PhD, ${ }^{1}$ Xiaoming Sheng, PhD, ${ }^{2}$ Christian M. Niedzwecki, DO, ${ }^{3}$ \\ Kimberly S. Bennett, MD, MPH, ${ }^{4}$ Denise C. Morita, MD, ${ }^{5}$ Brandon Zielinski, MD, PhD, ${ }^{5}$ and \\ Michelle E. Schober, MD, MS ${ }^{4}$ \\ ${ }^{1}$ Division of Pediatric Surgery, ${ }^{2}$ Department of Pediatrics, ${ }^{4}$ Division of Pediatric Critical Care, ${ }^{5}$ Division of Pediatric Neurology, and
} ${ }^{3}$ Department of Physical Medicine and Rehabilitation, University of Utah, Salt Lake City, Utah

\begin{abstract}
OBJECTIVE Traumatic brain injury (TBI) is the leading cause of acquired disability among children. Brain injury biomarkers may serve as useful diagnostic and prognostic indicators for TBI. Levels of ubiquitin C-terminal hydrolase-L1 (UCH-L1) and the 145-kDa alpha II-spectrin breakdown product (SBDP-145) correlate with outcome in adults after severe TBI. The authors conducted a pilot study of these biomarkers in children after severe TBI to inform future research exploring their utility in this population.
\end{abstract}

METHODS The levels of UCH-L1 and SBDP-145 were measured in serum, and UCH-L1 in CSF from pediatric patients after severe TBI over 5 days after injury. Both biomarkers were also measured in age-matched control serum and CSF.

RESULTS Adequate numbers of samples were obtained in serum, but not CSF, to assess biomarker temporal response profiles. Using patients with samples from all time points, UCH-L1 levels increased rapidly and transiently, peaking at 12 hours after injury. SBDP-145 levels showed a more gradual and sustained response, peaking at 48 hours. The median serum UCH-L1 concentration was greater in patients with TBI than in controls (median [IQR] = 361 [187, 1330] vs 147 $[50,241] \mathrm{pg} / \mathrm{ml}$, respectively; $p<0.001)$. Receiver operating characteristic (ROC) analysis revealed an AUC of 0.77. Similarly, serum SBDP-145 was greater in children with TBI than in controls (median [IQR] = $172[124,257]$ vs $69[40$, 99] pg/ml, respectively; $p<0.001$ ), with an ROC AUC of 0.85 . When only time points of peak levels were used for ROC analysis, the discriminability of each serum biomarker increased (AUC for UCH-L1 at 12 hours $=1.0$ and for SBDP-145 at 48 hours $=0.91)$. Serum and CSF UCH-L1 levels correlated well in patients with $\mathrm{TBI}(r=0.70, p<0.001)$.

CONCLUSIONS Findings from this exploratory study reveal robust increases of UCH-L1 and SBDP-145 in serum and UCH-L1 in CSF obtained from children after severe TBI. In addition, important temporal profile differences were found between these biomarkers that can help guide optimal time point selection for future investigations of their potential to characterize injury or predict outcomes after pediatric TBI.

https://thejns.org/doi/abs/10.3171/2018.4.PEDS17593

KEYWORDS traumatic brain injury; cerebrospinal fluid; serum; biomarkers; pediatric; trauma

$\mathrm{T}$ RAUMATIC brain injury (TBI) is a leading cause of death and disability among children..$^{5,11,16}$ Heterogeneity of TBI and highly variable outcomes complicate research and clinical care. Currently, bedside methods for prognostication and determination of the major pathophysiological mechanisms that drive injury at any given time are limited. These limitations pose challenges for research into potential therapies for this devastating prob- lem. In summary, clinically useful tools to improve classification and prognostication of TBI are needed.

Assessment of brain injury biomarkers holds great promise for better understanding the neurological response and recovery after TBI. Biomarker measurement may allow early and real-time bedside detection of pathophysiological processes activated after pediatric TBI. Such early detection could enable the design of targeted, patient-specific

ABBREVIATIONS AUC = area under the concentration-time curve; EVD = external ventricular drain; GCS = Glasgow Coma Scale; GFAP = glial fibrillary acidic protein; GOSE-P = Glasgow Outcome Scale Extended-pediatric version; ICP = intracranial pressure; ISS = injury severity score; PICU = pediatric intensive care unit; ROC = receiver operating characteristic; SBDP-145 = 145-kDa alpha II-spectrin breakdown product; TBI = traumatic brain injury; UCH-L1 = ubiquitin C-terminal hydrolase-L1.

SUBMITTED October 24, 2017. ACCEPTED April 17, 2018.

INCLUDE WHEN CITING Published online June 29, 2018; DOI: 10.3171/2018.4.PEDS17593. 
treatments to improve outcomes. The detection of brainderived markers in blood would be especially useful, as CSF is typically accessible in only a subset of patients with severe TBI. In recognition of the potential utility of biomarker measurement in pediatric TBI, the recently formed Pediatric TBI Common Data Elements Biospecimens and Biomarkers Workgroup published recommendations for pediatric biospecimen collection and analyses. ${ }^{1}$

Ubiquitin C-terminal hydrolase-L1 (UCH-L1) and the 145-kDa alpha II-spectrin breakdown product (SBDP-145) are potentially useful biomarkers for pediatric TBI. UCHL1 is an enzyme found almost exclusively in neurons. ${ }^{3}$ UCH-L1 is increased in both CSF and serum after TBI in adults, ${ }^{1,4,7,12,20,21,23-25,32}$ although less consistently after mild TBI. ${ }^{1,12,28,31}$ Alpha II-spectrin is a cytoskeletal protein primarily found in neuronal axons and dendrites. This protein is broken down into spectrin breakdown products of differing molecular weights. SBDP-145, a 145-kDa protein formed by calpain-mediated cleavage of spectrin, is a marker of necrotic cell death process activation. ${ }^{18,26,33}$ SBDP-145 levels in CSF are increased after severe TBI in adults. ${ }^{9,10,13,22,27}$ Previous studies have found both UCH-L1 and SBDP-145 to be elevated in serum after pediatric TBI, however, samples were collected at only a single, early time point after injury. ${ }^{2,19}$

The objective of our study was to characterize the temporal profile of SBDP-145 and UCH-L1 levels in serum, and UCH-L1 in CSF, in children after severe TBI. In accordance with the recommendations made by the Pediatric TBI Common Data Elements Biospecimens and Biomarkers Workgroup, we categorized specimens by time after injury, and included age-appropriate controls to address potential developmental influences on biomarker expression.

\section{Methods}

\section{Study Population and Sample Collection}

A prospective, observational pilot study was conducted within the pediatric intensive care unit (PICU) of a regional pediatric Level I trauma center between September 2011 and October 2013. Approval to conduct this research was obtained from the University of Utah IRB. Patients 0-17 years old admitted to the PICU were eligible for the study if they had severe TBI, defined as having known blunt head trauma resulting in an abnormal head CT scan and a need for mechanical ventilation for more than 24 hours from admission. Guardians were approached for consent within 12 hours of patient admission to the PICU. Patients were excluded if consent was unobtainable or denied, if they had an isolated epidural hematoma, or if the neurosurgical service deemed their injury to be nonsurvivable. Blood and CSF were collected at up to 5 time points $(12,24,72,96$, and 120 hours) after injury. Blood was collected via an existing venous or arterial line, with volumes limited to no more than a total of $10 \mathrm{ml}$ for the 5 collections. CSF was obtained only from those patients who already had an external ventricular drain (EVD) placed per standard care, and only while the EVD was still required for clinical management. Control samples were collected for each of four age brackets (less than 2 years, age 2-6, 7-12, and greater than 12 years old) from patients undergoing routine blood laboratory diagnostic testing for medical conditions unrelated to any neurological disorder and from whom there was no history of neurological injury. Similarly, samples for control CSF were obtained from patients undergoing routine lumbar puncture (oncology or emergency medicine patients) and were negative for infection and/or malignancy. These patients had no history of neurological injury.

Blood samples were allowed to clot for up to $30 \mathrm{~min}-$ utes and then centrifuged at 3500 RPM for 6 minutes. Supernatant (serum) was removed and stored at $-80^{\circ} \mathrm{C}$ until analysis. CSF was centrifuged at $3500 \mathrm{RPM}$ at $4^{\circ} \mathrm{C}$ for 10 minutes, and the supernatant was removed and stored at $-80^{\circ} \mathrm{C}$. Biomarkers were measured using a previously described sandwich enzyme-linked immunosorbent assay method (colorimetric format for UCH-L1, chemiluminescent format for SBDP-145) at Banyan Biomarkers Inc. ${ }^{17}$ SBDP-145 levels were not measured in CSF, as there were no validated, reliable assay methods for measurement in CSF available at the time. Control samples with concentrations below the limit of detection were assigned a value of half the concentration of the lower limit of detection for the respective assay (29\% of serum UCH-L1, $40 \%$ of CSF $\mathrm{UCH}-\mathrm{L} 1$, and $0 \%$ of serum SBDP-145 samples).

\section{Statistical Analysis}

Distributions of study variables were assessed using descriptive statistics. Differences between two groups were tested using the Wilcoxon rank-sum test. The Pearson correlation coefficient or Spearman rank correlation was used to measure correlations as appropriate. Area under the concentration-time curve (AUC) was calculated from time 0 to the maximum observed time using the trapezoidal rule. Linear mixed-effect models were utilized to examine time-course profiles of biomarker levels after TBI, while the receiver operating characteristic (ROC) was employed to assess discrimination between TBI and control subjects. Statistical differences were considered significant if the probability of a type I error was $<5 \%$. Study data were analyzed using SAS (version 9.4) or Stata (version 12.1).

\section{Results}

We collected 82 serum samples from the 19 enrolled patients with TBI, with a mean of 4.3 samples per patient. Eleven patients had serum samples for all 5 study time points. Not all patients had EVDs placed, and EVD duration was variable. As a result, we collected 38 CSF samples from 10 of the 19 patients with TBI, with a mean of 2 per patient, and only 4 patients had CSF samples from all 5 study time points. Therefore, temporal analyses were restricted to serum in the 11 patients with complete time points. All other analyses of biomarker parameters incorporated data from total serum and CSF samples collected. The number of serum samples obtained per patient was correlated with the number of CSF samples obtained per patient $(\mathrm{p}=0.006)$.

As shown in Table 1, the ages of the 19 patients ranged from 24 weeks to 15.7 years, with a median of 8.15 years ( $n=4$ for the three lower-age brackets and $n=7$ for those older than 12 years). Motor vehicle and auto-pedestrian 
TABLE 1. Patient and injury characteristics $(n=19)$

\begin{tabular}{lc}
\hline & Value \\
\hline Median age, yrs (range) & $8.15(0.47-15.69)$ \\
\hline Females, $\mathrm{n}(\%)$ & $7(37)$ \\
\hline Mechanism of injury, $\mathrm{n}(\%)$ & $9(47)$ \\
\hline Motor vehicle* & $5(26)$ \\
\hline Auto-pedestrian & $2(11)$ \\
\hline Nonaccidental (inflicted) & $2(11)$ \\
\hline Struck† & $1(5)$ \\
\hline Fall (snowboarding) & $5(1-6)$ \\
\hline Median best GCS motor score (range) $\ddagger$ & $18(95)$ \\
\hline Intracranial hemorrhage, $\mathrm{n}(\%)$ & $4(3-5)$ \\
\hline Median highest head AIS (range) & $7(37)$ \\
\hline Isolated head injury, $\mathrm{n}(\%) \S$ & $29(14-57)$ \\
\hline Median ISS (range) & $6(1-11)$ \\
\hline Outcomes & $7(2-15)$ \\
\hline Median days on ventilator (range) & $21(6-33)$ \\
\hline Median PICU LOS (range) & $3(16)$ \\
\hline Median hospital LOS (range)ף & $3(1-8)$ \\
\hline Deaths, $\mathrm{n}(\%)$ &
\end{tabular}

AIS = abbreviated injury score; LOS = length of stay.

* Includes car collisions $(n=5)$, all-terrain vehicle accidents $(n=2)$, motorcycle accident $(n=1)$, sledding behind car $(n=1)$.

$\dagger$ Includes struck by falling TV $(n=1)$ and kicked by horse $(n=1)$.

$\ddagger$ Using the highest score obtained in the first 6 hours after hospital admission in the absence of paralytics $(n=15)$.

$\S$ Having an AIS score no greater than 1 for any other body region.

Tी Sixteen cases, excluding 3 deaths.

${ }_{* \star}$ Score at 6 months after injury $(n=14)$.

accidents were the most common causes of injury. Most patients suffered injuries to other body regions in addition to head injury, with a median injury severity score (ISS) of 29. Intracranial hemorrhage was present in all but 1 case. Fourteen patients received either an intracranial pressure
(ICP) monitor $(\mathrm{n}=12)$ and/or an EVD $(\mathrm{n}=10)$. Patients with ICP monitoring, as well as 3 others, received hyperosmolar therapy in the form of hypertonic saline alone (n $=12$ ) or hypertonic saline and mannitol $(n=17)$. Fourteen of the 19 patients were assessed using the pediatric version of the Glasgow Outcome Scale-Extended (GOSE-P) at 6 months after injury (5 were lost to follow-up). Scores ranged from 1 (upper good recovery) to 8 (death; $n=3$ ), with a median score of 3 (upper moderate disability).

Using all available samples, serum UCH-L1 levels in patients with TBI were greater than those in controls (median $[\mathrm{IQR}]=361[187,1330]$ vs $147[50,241] \mathrm{pg} / \mathrm{ml}$, respectively; $\mathrm{p}<0.001$ ). Serum SBDP-145 showed a similar overall magnitude of increase over controls (median [IQR] $=172[124,257]$ vs $69[40,99] \mathrm{pg} / \mathrm{ml}$, respectively; $\mathrm{p}<$ $0.001)$. ROC analysis revealed an AUC of 0.77 (95\% CI 0.67-0.85) for serum UCH-L1, and an AUC of 0.85 (95\% CI 0.76-0.91) for serum SBDP-145.

As shown in Fig. 1, serum levels of UCH-L1 changed over time. UCH-L1 increased rapidly but transiently after TBI, peaking at the 12 -hour time point $(\mathrm{p}<0.001)$, falling back to control levels by 120 hours after injury. In contrast, SBDP-145 levels showed a more gradual and sustained increase, with the highest levels occurring at the 48-hour time point $(\mathrm{p}=0.011)$, and remaining different from controls at 120 hours after injury. When examining only peak concentrations, serum UCH-L1 reached greater levels than SBDP-145 (median 1630, range 448-3281, vs median 300 , range $173-431 \mathrm{pg} / \mathrm{ml}$, respectively; $\mathrm{p}<0.001$ ), and a higher magnitude increase over median control levels than SBDP-145 (11.1-fold vs 4.3-fold, respectively). When utilizing only those samples that were obtained at time points of peak response (12 hours for UCH-L1 and 48 hours for SBDP-145), the ability of these serum biomarkers to discriminate TBI from control subjects increased (AUC for UCH-L1 = 1.0 [95\% CI 0.88-1.0], and for SBDP$145=0.91[95 \%$ CI $0.77-0.98])$.

CSF UCH-L1 levels correlated well with serum levels $(r=0.70, p<0.001)$. Trend analysis demonstrated that the time course of serum UCH-L1 was similar to that of
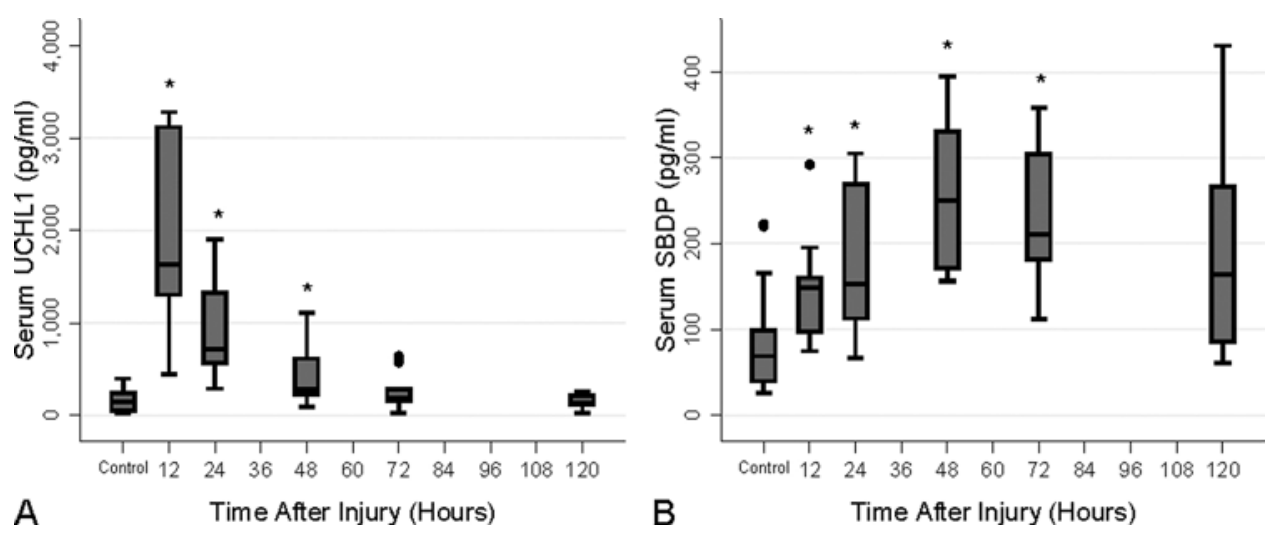

FIG. 1. Serum UCH-L1 (A) and SBDP-145 (B) levels over time after injury in patients with TBI. Both A and B represent results from the 11 patients for whom samples were obtained at all 5 study time points. The box plot whiskers for each biomarker depict 1.5 times the IQR from the nearest quartile (Tukey's method), while the box encompasses the upper and lower quartiles, and the bar the median value. Control values for $A$ and $B$ constructed using all the control samples obtained. *Statistically significant difference from control $(p<0.05)$. 

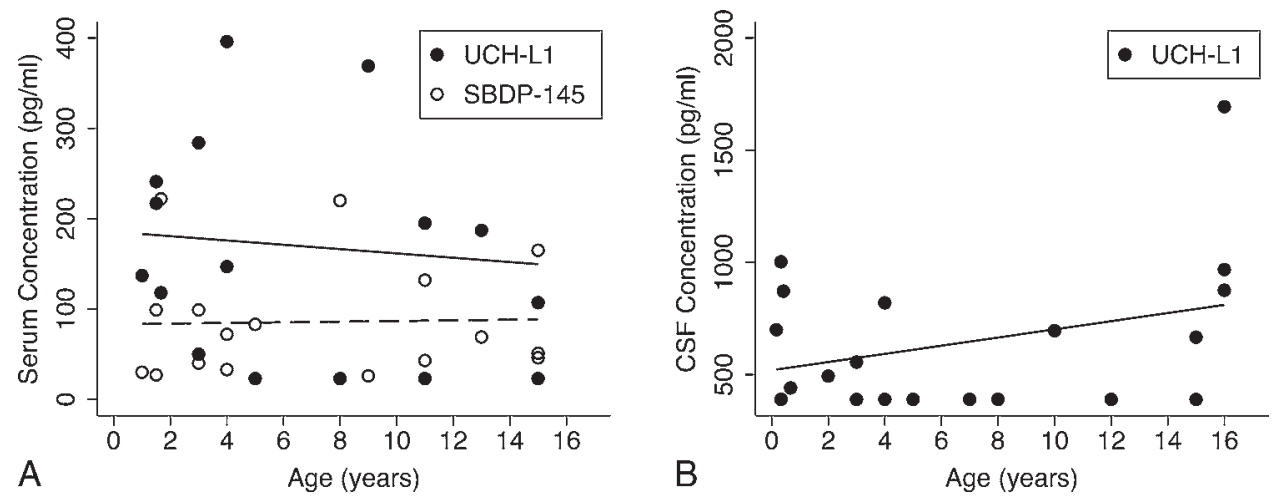

FIG. 2. UCH-L1 and SBDP-145 levels by age in control subjects. A: Solid dots represent serum UCH-L1 values, and circles represent serum SBDP-145 values, from noninjured control subjects $(n=17)$. Solid line represents a linear regression fitted to the UCH-L1 values $\left(p=0.697, R^{2}=0.010\right)$, and dashed line represents a linear regression fitted to the SBDP-145 values $(p=0.910$, $\left.R^{2}<0.001\right)$. B: Solid dots represent CSF UCH-L1 values from noninjured control subjects $(n=20)$. The line represents a linear regression fitted to the values $\left(p=0.148 ; R^{2}=0.113\right)$.

CSF UCH-L1, with levels peaking early $(\mathrm{p}=0.01$; data not shown). CSF UCH-L1 had a greater magnitude increase relative to controls than did serum UCH-L1 (CSF UCHL1 increased 6.4-fold: median 3372 [IQR 1209, 13553] vs 525 [IQR 390, 846] pg/ml, p < 0.001; serum UCH-L1 increased 2.5-fold [see above]).

A total of 17 control serum samples were obtained (n $=4$ for all age brackets, except $n=5$ for those aged 2-6 years), as well as 20 control CSF samples ( $n=5$ per age bracket), with overall median ages matching that of the TBI cohort. We did not observe differences in UCH-L1 or SBDP-145 concentrations between the four pediatric age groups in serum or in CSF, nor did levels appear to change with age across the entire cohort (Fig. 2; $p>0.5$ for rank correlation, and $p>0.1$ for linear correlation, for any of the 3 biomarker-biofluid combinations).

\section{Discussion}

Findings from this pilot study provide new insights into the response profiles of two candidate biomarkers for pediatric TBI, UCH-L1 and SBDP-145. Serum UCH-L1 and SBDP-145 levels increased robustly in children after severe TBI. Trend analyses revealed temporal response differences. Serum UCH-L1 levels display a rapid albeit transient increase after TBI, falling back to control levels by 120 hours. In contrast, serum SBDP-145, although significantly increased at 12 hours, follows a more gradual and sustained increase and peaks around 48 hours, but remains high through 120 hours after TBI.

To our knowledge, this is the first study to assess serum SBDP-145 levels over time after TBI in either adults or children. Berger et al. reported increased serum SBDP145 levels in pediatric patients with TBI relative to controls, but samples were limited to 1 time point (as soon as possible after arrival to the hospital), and were obtained from patients of variable TBI severity. ${ }^{2}$ We found that serum SBDP-145 levels peaked at about 48 hours after TBI. Previous findings on the temporal course of SBDP-145 levels are limited to CSF studies in adult patients, which showed that CSF levels peaked at approximately 2-3 days after severe TBI. ${ }^{9,10,13}$ Two other studies in which CSF was obtained more frequently (every 3-6 hours in the first 48 hours) showed a marked peak in SBDP-145 in the first 24 hours after injury, followed by a gradual decline toward control levels over several days.22,27 CSF SBDP-145 levels were not measured in the present study. We previously showed that rat pup brain tissue SBDP-145 levels peaked in the first 48 hours after injury in a well-established model of pediatric TBI. Based on our experimental findings, we speculate that SBDP-145 levels in pediatric CSF would peak in the first few days after TBI, as they had in the adult studies, but not necessarily in the first 24 hours.

We are unaware of any published work reporting serum UCH-L1 levels over time following pediatric TBI. Berger et al. found that serum UCH-L1 levels (measured at a single time point soon after hospital arrival) in pediatric patients with moderate or severe TBI were increased relative to control values. ${ }^{2} \mathrm{~A}$ subsequent study by this group, using a similar paradigm, reported comparable findings..$^{19}$ However, Rhine et al. ${ }^{31}$ did not find increased serum levels in teenagers at a single time-point soon after mild TBI, compared to orthopedic controls. Studies in adult patients with TBI consistently demonstrate that serum and CSF UCH-L1 levels are highest at early time points after TBI. ${ }^{4,7,20,21,25,29}$ Of note, the FDA recently approved using a serum UCH-L1 and glial fibrillary acidic protein (GFAP) panel (the Banyan "Brain Trauma Indicator," Banyan Biomarkers, Inc.) in adult patients within 12 hours after mild TBI to help predict brain injury detectable by CT. ${ }^{14}$ Our findings support future research using serum UCH-L1 in children within the same timeframe (12 hours after injury).

We found that serum UCH-L1 and SBDP-145 exhibited modest discrimination between injured and control patients when utilizing all TBI samples, regardless of time from injury. Given the differential effect of time after injury on levels of these biomarkers, it was not surprising to find that discriminability improved when using only early time points for UCH-L1, and later time points for SBDP145 , as reported previously for UCH-L1 in adults..$^{12,20,24,25}$ This was especially the case for UCH-L1 because serum levels returned to control levels at later time points after injury. 
This study has several limitations. We achieved the sample number needed to assess biomarker temporal response profiles in serum but not CSF. The final sample size in serum levels, however, still sufficed to determine whether these biomarkers were significantly higher in patients with severe TBI versus control subjects, and whether levels showed time-dependent changes according to linear mixed-effects modeling.

Another potential limitation is that lumbar, not ventricular, CSF was used for control samples. Comparisons to ventricular CSF from patients with TBI could be thus confounded should a ventricular-lumbar gradient for UCH-L1 exist. Two publications address this issue. ${ }^{6,30}$ Unfortunately, ventricular samples from children without brain pathology are difficult to obtain in a reliable manner. Using ventricular samples from children with brain pathology is not ideal. For example, production of UCH-L1 by a brain tumor, or by brain injury secondary to pressure or vascular insults, could confound the results and render the sample unsuitable as a control.

Similar to the results in a previous study in adult patients with TBI, UCH-L1 in serum and CSF correlated fairly well in this study, suggesting that serum UCH-L1 could serve as a proxy for CSF levels.? The difference in serum concentrations between TBI and controls was smaller than that observed in CSF (ROC AUC was 0.77 vs 0.85 , respectively), suggesting that UCH-L1 levels in serum may have less discriminatory capability than in CSF, as would be expected.

This study also contributes data to help address the important question of whether developmental age affects biomarker levels in children. We did not find any differences in levels across the four pediatric age groups studied, suggesting that neither UCH-L1 nor SBDP-145 vary in serum, or UCH-L1 in CSF, as a function of age, although low statistical power (due to small sample sizes of the age groups) could limit detection of any real differences. We did not observe a trend when we plotted biomarker levels against age for the entire control cohort. Mondello et al. noted that serum UCH-L1 levels were high in control infants compared to samples from all other age groups in their study. ${ }^{19}$ They did not specify the actual levels nor how they calculated results from samples that may have fallen below detection limits (a common occurrence in uninjured controls). While we did not find higher $\mathrm{UCH}-$ L1 levels in our youngest age bracket of controls (those younger than 2 years), small sample size could have limited our ability to detect infant-specific differences within our youngest age bracket.

We did not observe any statistically significant relationships between biomarker levels and injury characteristics or outcomes. One reason may be that we had not powered our study for this goal. In addition, the narrow range of values available for the primary prognostic and outcome measures (the best motor Glasgow Coma Scale [GCS] score and the GOSE-P, respectively) adds another statistical limitation. Finally, because study patients were restricted to severe TBI, the narrower range of outcomes (relative to that possible if less severe forms of TBI are included) further limits the ability to detect associations between variables.

\section{Conclusions}

This exploratory study provides novel information on the temporal aspects of UCH-L1 and SBDP-145 responses in serum after pediatric TBI, and on their developmental expression in uninjured children. We observed a rapid decline from an early peak response in UCH-L1 levels after TBI, suggesting that UCH-L1 would likely have low sensitivity or prognostic power if measured days after injury rather than hours. ${ }^{8}$ In contrast, the temporal profile observed for SBDP-145 levels suggests that measuring only SBDP-145 at an early time point would be inadequate for estimating TBI severity or for screening for mild TBI. Future studies to determine the suitability of a combined biomarker assessment for either detecting or characterizing brain injury, using larger sample sizes, are warranted. Our findings support a future trial in children to study whether serum UCH-L1 levels obtained within 12 hours after TBI, combined or not with GFAP levels, may help predict brain lesions detectable by CT scans as has been now shown in adult patients. ${ }^{14}$ In addition, such studies could further investigate whether those biomarkers with sustained responses, such as SBDP-145, could serve to help monitor recovery or the impact of putative neuroprotective therapies.

\section{Acknowledgments}

This study was supported by a Primary Children's Hospital Foundation Integrated Science Award (all authors) and by the University of Utah Clinical and Translational Sciences (grant no. CTSA 5UL1RR025764-01; Dr. Metzger). Study data were collected and managed using REDCap (Research Electronic Data Capture) tools hosted at the University of Utah. ${ }^{15}$

\section{References}

1. Berger RP, Beers SR, Papa L, Bell M: Common data elements for pediatric traumatic brain injury: recommendations from the biospecimens and biomarkers workgroup. J Neurotrauma 29:672-677, 2012

2. Berger RP, Hayes RL, Richichi R, Beers SR, Wang KK: Serum concentrations of ubiquitin C-terminal hydrolase-L1 and $\alpha$ II-spectrin breakdown product $145 \mathrm{kDa}$ correlate with outcome after pediatric TBI. J Neurotrauma 29:162-167, 2012

3. Bishop P, Rocca D, Henley JM: Ubiquitin C-terminal hydrolase L1 (UCH-L1): structure, distribution and roles in brain function and dysfunction. Biochem J 473:2453-2462, 2016

4. Blyth BJ, Farahvar A, He H, Nayak A, Yang C, Shaw G, et al: Elevated serum ubiquitin carboxy-terminal hydrolase L1 is associated with abnormal blood-brain barrier function after traumatic brain injury. J Neurotrauma 28:2453-2462, 2011

5. Bowman SM, Bird TM, Aitken ME, Tilford JM: Trends in hospitalizations associated with pediatric traumatic brain injuries. Pediatrics 122:988-993, 2008

6. Brandner S, Thaler C, Lewczuk P, Lelental N, Buchfelder M, Kleindienst A: Neuroprotein dynamics in the cerebrospinal fluid: intraindividual concomitant ventricular and lumbar measurements. Eur Neurol 70:189-194, 2013

7. Brophy GM, Mondello S, Papa L, Robicsek SA, Gabrielli A, Tepas J III, et al: Biokinetic analysis of ubiquitin C-terminal hydrolase-L1 (UCH-L1) in severe traumatic brain injury patient biofluids. J Neurotrauma 28:861-870, 2011

8. Brophy GM, Pineda JA, Papa L, Lewis SB, Valadka AB, Hannay HJ, et al: $\alpha$ II-Spectrin breakdown product cerebrospinal fluid exposure metrics suggest differences in cellular 
injury mechanisms after severe traumatic brain injury. $\mathbf{J}$ Neurotrauma 26:471-479, 2009

9. Cardali S, Maugeri R: Detection of alphaII-spectrin and breakdown products in humans after severe traumatic brain injury. J Neurosurg Sci 50:25-31, 2006

10. Chen S, Shi Q, Zheng S, Luo L, Yuan S, Wang X, et al: Role of $\alpha$-II-spectrin breakdown products in the prediction of the severity and clinical outcome of acute traumatic brain injury. Exp Ther Med 11:2049-2053, 2016

11. Dewan MC, Mummareddy N, Wellons JC III, Bonfield CM: Epidemiology of global pediatric traumatic brain injury: qualitative review. World Neurosurg 91:497-509, 509.e1, 2016

12. Diaz-Arrastia R, Wang KK, Papa L, Sorani MD, Yue JK, Puccio AM, et al: Acute biomarkers of traumatic brain injury: relationship between plasma levels of ubiquitin Cterminal hydrolase-L1 and glial fibrillary acidic protein. J Neurotrauma 31:19-25, 2014

13. Farkas O, Polgár B, Szekeres-Barthó J, Dóczi T, Povlishock JT, Büki A: Spectrin breakdown products in the cerebrospinal fluid in severe head injury-preliminary observations. Acta Neurochir (Wien) 147:855-861, 2005

14. Food and Drug Administration: FDA authorizes marketing of first blood test to aid in the evaluation of concussion in adults. FDA.gov. February 14, 2018. (https://www.fda.gov/ NewsEvents/Newsroom/PressAnnouncements/ucm596531. htm) [Accessed May 7, 2018]

15. Harris PA, Taylor R, Thielke R, Payne J, Gonzalez N, Conde JG: Research electronic data capture (REDCap) - a metadata-driven methodology and workflow process for providing translational research informatics support. J Biomed Inform 42:377-381, 2009

16. Keenan HT, Bratton SL: Epidemiology and outcomes of pediatric traumatic brain injury. Dev Neurosci 28:256-263, 2006

17. Liu MC, Akinyi L, Scharf D, Mo J, Larner SF, Muller U, et al: Ubiquitin C-terminal hydrolase-L1 as a biomarker for ischemic and traumatic brain injury in rats. Eur J Neurosci 31:722-732, 2010

18. McGinn MJ, Kelley BJ, Akinyi L, Oli MW, Liu MC, Hayes RL, et al: Biochemical, structural, and biomarker evidence for calpain-mediated cytoskeletal change after diffuse brain injury uncomplicated by contusion. J Neuropathol Exp Neurol 68:241-249, 2009

19. Mondello S, Kobeissy F, Vestri A, Hayes RL, Kochanek PM, Berger RP: Serum concentrations of ubiquitin C-terminal hydrolase-L1 and glial fibrillary acidic protein after pediatric traumatic brain injury. Sci Rep 6:28203, 2016

20. Mondello S, Linnet A, Buki A, Robicsek S, Gabrielli A, Tepas J, et al: Clinical utility of serum levels of ubiquitin Cterminal hydrolase as a biomarker for severe traumatic brain injury. Neurosurgery 70:666-675, 2012

21. Mondello S, Papa L, Buki A, Bullock MR, Czeiter E, Tortella FC, et al: Neuronal and glial markers are differently associated with computed tomography findings and outcome in patients with severe traumatic brain injury: a case control study. Crit Care 15:R156, 2011

22. Mondello S, Robicsek SA, Gabrielli A, Brophy GM, Papa L, Tepas J, et al: $\alpha$ II-spectrin breakdown products (SBDPs): diagnosis and outcome in severe traumatic brain injury patients. J Neurotrauma 27:1203-1213, 2010

23. Papa L, Akinyi L, Liu MC, Pineda JA, Tepas JJ III, Oli MW, et al: Ubiquitin C-terminal hydrolase is a novel biomarker in humans for severe traumatic brain injury. Crit Care Med 38:138-144, 2010

24. Papa L, Brophy GM, Welch RD, Lewis LM, Braga CF, Tan $\mathrm{CN}$, et al: Time course and diagnostic accuracy of glial and neuronal blood biomarkers GFAP and UCH-L1 in a large cohort of trauma patients with and without mild traumatic brain injury. JAMA Neurol 73:551-560, 2016

25. Papa L, Lewis LM, Silvestri S, Falk JL, Giordano P, Brophy
GM, et al: Serum levels of ubiquitin C-terminal hydrolase distinguish mild traumatic brain injury from trauma controls and are elevated in mild and moderate traumatic brain injury patients with intracranial lesions and neurosurgical intervention. J Trauma Acute Care Surg 72:1335-1344, 2012

26. Pike BR, Flint J, Dutta S, Johnson E, Wang KK, Hayes RL: Accumulation of non-erythroid $\alpha$ II-spectrin and calpaincleaved $\alpha$ II-spectrin breakdown products in cerebrospinal fluid after traumatic brain injury in rats. J Neurochem 78:1297-1306, 2001

27. Pineda JA, Lewis SB, Valadka AB, Papa L, Hannay HJ, Heaton SC, et al: Clinical significance of $\alpha$ II-spectrin breakdown products in cerebrospinal fluid after severe traumatic brain injury. J Neurotrauma 24:354-366, 2007

28. Posti JP, Hossain I, Takala RS, Liedes H, Newcombe V, Outtrim J, et al: Glial fibrillary acidic protein and ubiquitin C-terminal hydrolase-L1 are not specific biomarkers for mild CT-negative traumatic brain injury. J Neurotrauma 34:1427-1438, 2017

29. Posti JP, Takala RS, Runtti H, Newcombe VF, Outtrim J, Katila AJ, et al: The levels of glial fibrillary acidic protein and ubiquitin C-terminal hydrolase-L1 during the first week after a traumatic brain injury: correlations with clinical and imaging findings. Neurosurgery 79:456-464, 2016

30. Reiber H, Peter JB: Cerebrospinal fluid analysis: diseaserelated data patterns and evaluation programs. J Neurol Sci 184:101-122, 2001

31. Rhine T, Babcock L, Zhang N, Leach J, Wade SL: Are UCHL1 and GFAP promising biomarkers for children with mild traumatic brain injury? Brain Inj 30:1231-1238, 2016

32. Siman R, Toraskar N, Dang A, McNeil E, McGarvey M, Plaum J, et al: A panel of neuron-enriched proteins as markers for traumatic brain injury in humans. J Neurotrauma 26:1867-1877, 2009

33. Zhang Z, Larner SF, Liu MC, Zheng W, Hayes RL, Wang KK: Multiple alphaII-spectrin breakdown products distinguish calpain and caspase dominated necrotic and apoptotic cell death pathways. Apoptosis 14:1289-1298, 2009

\section{Disclosures}

The authors report no conflict of interest concerning the materials or methods used in this study or the findings specified in this paper.

\section{Author Contributions}

Conception and design: Schober, Metzger, Niedzwecki, Bennett, Morita. Acquisition of data: Schober, Metzger, Niedzwecki, Bennett, Morita, Zielinski. Analysis and interpretation of data: Schober, Metzger, Sheng, Niedzwecki, Bennett, Morita. Drafting the article: Schober, Metzger. Critically revising the article: Schober, Metzger, Niedzwecki, Bennett, Morita. Reviewed submitted version of manuscript: Schober, Metzger, Niedzwecki, Bennett, Morita, Zielinski. Approved the final version of the manuscript on behalf of all authors: Schober. Statistical analysis: Metzger, Sheng. Administrative/technical/material support: Metzger. Study supervision: Schober, Metzger, Bennett.

\section{Supplemental Information}

\section{Previous Presentations}

Portions of this work were presented in abstract and poster form at the 12th Annual Neurocritical Care Society Meeting in Seattle, Washington, September 11-14, 2014.

\section{Correspondence}

Michelle E. Schober: University of Utah School of Medicine, Salt Lake City, UT. michelle.schober@hsc.utah.edu. 